\title{
ANÁlise da MADEIRA do Pinus oocarpa PARTE II - CARACTERIZAÇÃo ESTRUTURAL DA LIGNINA DE MADEIRA MOÍDA ${ }^{1}$
}

\author{
Sérgio Antônio Lemos de Morais², Evandro Afonso do Nascimento² e Dárley Carrijo de Melo ${ }^{3}$ \\ RESUMO - Este estudo caracterizou a Lignina de Madeira Moída (LMM) proveniente de Pinus oocarpa cultivado \\ na região do Cerrado brasileiro. A LMM foi isolada e analisada por meio das espectrometrias no infravermelho \\ com transformada de Fourier (IVTF), de ressonância magnética nuclear do próton e carbono-13 e por intermédio \\ de métodos químicos de análise por via úmida. ALMM apresentou uma fórmula mínima igual a $\mathrm{C}_{9} \mathrm{H}_{9,2} \mathrm{O}_{2,6}\left(\mathrm{OCH}_{3}\right)_{0,8}$ \\ e massas molares médias em massa $\left(\bar{M}_{w}\right)$ e numérica $\left(\bar{M}_{n}\right)$ de 3.969 e $1.133 \mathrm{Da}$, respectivamente. A LMM \\ dessa madeira se enquadra dentro das ligninas típicas de coníferas.
}

Palavras-chave: Pinus oocarpa, lignina e lignina de madeira moída.

\section{CHEMICAL ANALYSIS OF THE Pinus oocarpa WOOD.PART II - CHARACTERIZATION OF THE MILLED WOOD LIGNIN}

\begin{abstract}
This work presents the characterization of the milled wood lignin (MWL) of the Pinus oocarpa cultivated in the Brazilian cerrado. FTIR, carbon-13 and proton NMR spectroscopies as well as wet chemical methods were used. The established $C_{9}$ unit formula for $M W L$ was $\mathrm{C}_{9} \mathrm{H}_{9,2} \mathrm{O}_{2,6}\left(\mathrm{OCH}_{3}\right)_{0,8}$ and its relative molecular weights $\left(\bar{M}_{w}\right)$ and $\left(\bar{M}_{n}\right)$ were 3969 and 1133 Da, respectively. Pinus oocarpa MWL was typical of softwood lignins.
\end{abstract}

Key words: Pinus oocarpa, lignin, milled wood lignin.

\section{INTRODUÇÃO}

A madeira é constituída fundamentalmente pelos componentes macromoleculares celulose, hemicelulose e lignina, presentes em porcentuais variáveis. Uma simplificação indica que a celulose é um esqueleto envolvido por duas substâncias que funcionam como recheio (hemiceluloses ou polioses), sendo ambas envolvidas pela lignina, que atua como um cimento para dar rigidez ao conjunto.

A lignina, o segundo material mais abundante no reino vegetal, é uma substância química que confere rigidez à parede celular e atua como um composto de ligação da celulose e polioses, gerando uma estrutura resistente a impacto, compressão e dobra. Pelo decréscimo que causa na permeação de água por meio das paredes das células dos tecidos condutores do xilema, a lignina tem uma função importante no intrincado transporte interno de água, nutrientes e metabólitos (PHILIPP e D'ALMEIDA, 1988).

A lignina é um polímero natural proveniente da condensação desidrogenerativa de três álcoois precursores: trans-coniferílico, trans-sinapílico e $p$ cumarílico. Em madeiras de coníferas (moles) predominam, basicamente, subestruturas do tipo guaiacilpropano,

\footnotetext{
${ }^{1}$ Recebido em $1^{\circ} .09 .2003$ e aceito para publicação em 20.04.2005.

${ }^{2}$ Instituto de Química da Universidade Federal de Uberlândia - UFU, 38400-089 Uberlândia-MG. E-mail: <salemos@ ufu.br>.

${ }^{3}$ Faculdade de Engenharia Química da UFU.
} 
provenientes do álcool trans-coniferílico; em madeiras de folhosas (duras), existe uma mistura de quantidades significativas de subestruturas guaiacil e siringilpropano, resultantes da polimerização dos precursores transconiferílico e trans-sinapílico, respectivamente. Já em palhas e gramíneas, além de unidades guaiacílicas e siringílicas, encontram se subestruturas do tipo $p$ hidroxifenilpropano proveniente da polimerização do álcool $p$-cumarílico, todas em quantidades relevantes. A proporção molar dessas subestruturas na planta depende, principalmente, da espécie (CHEN, 1991).

O conhecimento dos componentes da estrutura química da lignina é de fundamental importância para seu posterior aproveitamento, assim como para a proteção do meio ambiente. As ligninas são obtidas em larga escala, em todo o mundo, como subproduto, principalmente, da indústria de polpação em que seu aproveitamento principal é como fonte de energia obtida pela queima, mas parte dela acaba sendo descartada como rejeito (MORAIS et al., 1993).

Existem diferentes tipos de extração de ligninas, e nenhum deles permite obtê-la como ela se encontra estruturalmente no vegetal, pois haverá sempre interferência entre os procedimentos de extração química e a estrutura da lignina in situ. Um bom método de extração deve eliminar os extrativos da madeira sem alterar significativamente a lignina. Os procedimentos que mais se adequam à extração envolvem a moagem em moinho de bolas, sob a atmosfera inerte, na presença ou ausência de solventes como tolueno, seguida da extração com clorofórmio, depois água e, finalmente, uma mistura de dioxano ou acetona aquosos. A lignina obtida é designada como Lignina de Madeira Moída (Milled Wood Lignin - MWL) (MORAIS et al., 1993).

Como não se encontrou na literatura nenhum estudo químico aprofundado acerca da lignina de madeira do Pinus oocarpa, o presente trabalho teve como objetivos isolar a lignina de madeira moída (LMM) e caracterizar os principais grupos funcionais presentes, identificar as principais unidades estruturais por métodos espectrométricos de análise (FTIR, RMN de ${ }^{1} \mathrm{He}{ }^{13} \mathrm{C}$ ) e estabelecer uma fórmula mínima.

$\mathrm{Na}$ parte I deste trabalho foram estudados os constituintes macromoleculares e extrativos da madeira de Pinus oocarpa.

\section{MATERIAL E MÉTODOS}

\subsection{Madeira de Pinus oocarpa}

A árvore foi proveniente de um reflorestamento da região de Sacramento, MG, tinha oito anos de idade e foi cortada à altura do peito. $\mathrm{O}$ disco resultante mediu $15,0 \mathrm{~cm}$ de diâmetro e 2,0 cm de espessura. Depois de descascado, ele foi picado em cavacos e moído em moinho de lâmina até a granulometria próxima de 1,0 $\mathrm{mm}$. A madeira obtida foi moída em um moinho de bolas por duas semanas e peneirada, recolhendo-se a fração entre 40-60 mesh.

\subsection{Isolamento da Lignina de Madeira Moída (LMM)}

A fração de madeira moída com granulometria entre 40 e 60 mesh foi previamente submetida à extração com etanol: tolueno $(1: 2, \mathrm{v} / \mathrm{v})$ para eliminação dos extrativos. A LMM foi extraída e purificada de acordo com o procedimento descrito por Lundquist (1992).

\subsection{Caracterização da LMM}

\subsubsection{Análise Elementar}

Os teores de carbono, hidrogênio e nitrogênio foram determinados no Laboratório da Central Analítica da USP - São Paulo. O valor do oxigênio foi calculado por diferença.

\subsubsection{Acetilação}

A acetilação foi realizada de acordo com o método descrito por Chen (1992a).

\subsubsection{Ressonância Magnética Nuclear do Carbono 13 (RMN de ${ }^{13} \mathrm{C}$ ) e Hidrogênio (RMN de ${ }^{1} \mathrm{H}$ )}

Os espectros em RMN de ${ }^{13} \mathrm{C}$ foram obtidos na freqüência de $100,6 \mathrm{MHz}$, usando-se $\mathrm{CDCl}_{3}$ como solvente, seqüência de pulso ZGPG 30, ângulo de $30^{\circ}$ para o pulso principal e aquisição de pulso a 1,0 s, com o acúmulo de 10.000 espectros.

Para os espectros em RMN de ${ }^{1} \mathrm{H}$, foi usada uma freqüência de $400 \mathrm{MHz}$, e a aquisição de pulso foi a cada $0,1 \mathrm{~s}$, sendo acumulados 500 espectros.

\subsubsection{Espectros no Infravermelho}

Os espectros no infravermelho foram obtidos em um espectrofotômetro da firma Shimadzu, modelo FTIR, série 8000 . E 1,0 mg da amostra foi misturado e triturado com 99,0 mg de $\mathrm{KBr}$, por um período de $20 \mathrm{~min}$; depois, 
a mistura foi pastilhada e introduzida no espectrofotômetro.

\subsubsection{Reação de Oximação e Determinação de Carbonilas Totais}

Procedeu-se à reação de oximação de acordo com o método proposto por Kröger et al. (1965). Para calcular o teor de carbonilas na macromolécula de lignina, utilizaram-se os valores da análise de CHN da LMM antes e depois da reação de oximação (MORAIS, 1992).

O cálculo da porcentagem de carbonilas $(\mathrm{C}=\mathrm{O})$ foi realizado por meio da equação 1 (KRÖGER et al.,1965).

$$
\% \mathrm{C}=\mathrm{O}=\frac{186,2\left(\% \mathrm{~N}_{1}-\% \mathrm{~N}\right)}{93,4-\left(\% \mathrm{~N}_{1}-\% \mathrm{~N}\right)}
$$

em que:

$\% \mathrm{~N}_{1}=$ teor de nitrogênio na lignina após a reação de oximação; e

$\% \mathrm{~N}=$ teor de nitrogênio na lignina antes da reação de oximação.

A partir desse valor, calculou-se o teor molar de carbonilas por mol de metoxilas $\left(\mathrm{C}=\mathrm{O} / \mathrm{OCH}_{3}\right)$, utilizando a equação 2 .

$$
\mathrm{C}=\mathrm{O} / \mathrm{OCH}_{3}=\frac{31(\% \mathrm{C}=\mathrm{O})}{28\left(\% \mathrm{OCH}_{3}\right)}
$$

A quantidade molar por unidade $\mathrm{C}_{9}\left(\mathrm{C}=\mathrm{O} / \mathrm{C}_{9}\right)$ foi determinada pela equação 3 .

$$
\mathrm{C}=\mathrm{O} / \mathrm{C}_{9}=\left(\mathrm{C}=\mathrm{O} / \mathrm{OCH}_{3}\right) \times\left(\mathrm{n}^{\mathrm{o}} \mathrm{OCH}_{3} / \mathrm{C}_{9}\right)
$$

\subsubsection{Determinação dos Grupos Metoxílicos}

Para a determinação do teor de grupos metoxílicos, foi utilizado o procedimento modificado de Zeizel-VieböckSchwappach (ZAKIS et al., 1994).

\subsubsection{Determinação da Fórmula Mínima}

Utilizando os valores das porcentagens de carbono, hidrogênio e nitrogênio, obtidos por meio da análise de CHN e do teor de grupos metoxílicos, determinouse a fórmula mínima para a lignina. Segundo Freudenberg e Neish (1968), a fórmula mínima de ligninas pode ser representada pela fórmula geral $\mathrm{C}_{9} \mathrm{H}_{\mathrm{X}} \mathrm{O}_{\mathrm{Y}}\left(\mathrm{OCH}_{3}\right)_{\mathrm{Z}}$, em que $\mathrm{X}, \mathrm{Y}$ e $\mathrm{Z}$ são dados pelas seguintes equações:

$$
\mathrm{X}=\frac{277(\% \mathrm{H})-27\left(\% \mathrm{OCH}_{3}\right)}{2,584(\% \mathrm{C})-\left(\% \mathrm{OCH}_{3}\right)}
$$

$$
\begin{aligned}
& \mathrm{Y}=\frac{17,45(\% \mathrm{O})-9\left(\% \mathrm{OCH}_{3}\right)}{2,584(\% \mathrm{C})-\left(\% \mathrm{OCH}_{3}\right)} \\
& \mathrm{Z}=\frac{9\left(\% \mathrm{OCH}_{3}\right)}{2,584(\% \mathrm{C})-\left(\% \mathrm{OCH}_{3}\right)}
\end{aligned}
$$

\subsubsection{Determinação de Grupos Hidroxila}

O teor de hidroxilas totais foi estimado por meio dos teores de carbono calculados antes e depois da acetilação, equação 7 (LUNDQUIST, 1979).

$$
\% \mathrm{OH}_{\text {total }}=\frac{40,5(\% \mathrm{C}-\% \mathrm{C})}{(\% \mathrm{C}-57,14)}
$$

em que:

$\% \mathrm{C}=$ porcentagem de carbono na lignina antes da reação de acetilação; e

$\% \mathrm{C}^{\prime}=$ porcentagem de carbono na lignina acetilada .

A quantidade molar de hidroxilas totais por unidade $\mathrm{C}_{9}$ foi calculada pela equação 8 .

As hidroxilas fenólicas e alifáticas foram estimadas a partir do valor de hidroxilas totais e da intensidade de prótons provenientes desses grupos no espectro de ressonância magnética nuclear protônica (RMN de $\left.{ }^{1} \mathrm{H}\right)$.

\subsubsection{Distribuição de Massas Molares}

A distribuição de massas molares relativas, média em massa () e massa molar média em número (), foi feita por meio da análise de cromatografia por permeação de gel (CPG).

\subsubsection{Reação de Oxidação com Nitrobenzeno}

Para a oxidação com nitrobenzeno foi utilizado um reator Parr de 25,0 mL. Foram colocados 25,0 mg de lignina, 9,0 mL de solução de $\mathrm{NaOH}\left(2,5 \mathrm{~mol} \mathrm{dm}^{-3}\right)$ e $1,0 \mathrm{~mL}$ de nitrobenzeno no reator, que foi fechado e deixado a $170{ }^{\circ} \mathrm{C}$ durante três horas, para que a reação pudesse ocorrer. O produto oxidado foi purificado e o derivado sililado, obtido através da reação com BSTFA, de acordo com o procedimento descrito por Chen (1992b). Os produtos foram identificados e quantificados por cromatografia gasosa acoplada à espectrometria de massas (CG-EM).

R. Árvore, Viçosa-MG, v.29, n.3, p.471-478, 2005 


\section{RESULTADOS E DISCUSSÃO}

A redução de contaminantes alifáticos foi uma das dificuldades encontradas ao isolar a LMM do Pinus oocarpa. Vários procedimentos foram testados, e o que se mostrou mais adequado para o isolamento da LMM foi o descrito por Lundquist (1992).

As análises mais indicativas da reprodutibilidade de uma lignina são os teores de grupos metoxilas e CHN (MORAIS et al., 1993). Na Tabela 1, apresentase a análise dos conteúdos de carbono, hidrogênio, nitrogênio e oxigênio da LMM, lignina de madeira moída acetilada (LMM-Ac), LMM após a reação de oximação (LMM-Ox) e a porcentagem de grupos metoxilas.

Dessa forma, utilizando os valores da análise de $\mathrm{CHN}$ e o conteúdo de $\mathrm{OCH}_{3}$, determinou-se a fórmula mínima para a LMM do Pinus oocarpa: $\mathrm{C}_{9} \mathrm{H}_{9,2} \mathrm{O}_{2,6}(\mathrm{OCH} 3)_{0,8}$. Assim, a unidade $\mathrm{C}_{9}$ da lignina do Pinus oocarpa apresenta massa molar de 183,6 Da. A quantidade de $\mathrm{OCH}_{3} / \mathrm{C}_{9}$, equivalente a 0,8 para essa lignina, foi, portanto, abaixo de 1,0, indicando a presença de unidades $p$-hidroxifenilpropano, que são desprovidas de metoxilas, compondo a macromolécula dessa lignina.

Ligninas contêm grupos carbonilas que podem estar conjugados ou não com o anel aromático. Utilizando os valores de porcentagem de nitrogênio antes e depois da reação de oximação (Tabela 1), calculou-se uma porcentagem de carbonila igual a 1,62\%. A partir desse valor, determinaram-se o teor molar de carbonilas por mol de metoxilas e a quantidade molar por unidade $\mathrm{C}_{9}\left(\mathrm{C}=\mathrm{O} / \mathrm{C}_{9}\right)$, que resultaram em 0,13 e 0,10 , respectivamente. Assim, em 100 unidades $\mathrm{C}_{9}$ estão presentes 10 grupos carbonila.

Tabela 1 - Resultados da análise elementar e conteúdo de grupos metoxila para LMM, LMM-Ac e LMMOx do Pinus oocarpa

Table 1 - Elemental composition and methoxyl groups content of $M W L$, acetylated $M W L$ and oximated $M W L$ from Pinus oocarpa

\begin{tabular}{lccc}
\hline & \multicolumn{3}{c}{ Composição (\%) } \\
\cline { 2 - 4 } & LMM & LMM-Ac & LMM-Ox \\
\hline $\mathrm{C}$ & 63,44 & 62,11 & 57,23 \\
$\mathrm{H}$ & 6,33 & 5,47 & 6,07 \\
$\mathrm{~N}$ & 0,99 & 0,50 & 1,80 \\
$\mathrm{O}$ & 29,24 & 31,92 & 34,90 \\
OCH3 & 13,61 & - & - \\
\hline
\end{tabular}

As hidroxilas têm papel importante no tocante às propriedades da macromolécula de lignina, como solubilidade e propriedades adesivas. A quantidade de hidroxilas totais na macromolécula de lignina é estimada usando os valores do teor de carbono na molécula antes e depois da reação de acetilação (MORAIS, 1992). Empregando os valores porcentuais de carbono da LMM antes e depois da reação de acetilação (Tabela 1), encontrou-se um teor de hidroxilas totais de $8,55 \%$, o que equivale a 0,92 de hidroxila total por unidade monomérica $\left(\mathrm{C}_{9}\right)$. Esse resultado indica que em 100 unidades $\mathrm{C}_{9}$ de lignina estão presentes 92 grupos de hidroxilas fenólicas e alifáticas.

As propriedades de ligninas são mais bem compreendidas se estimar a massa molar ou sua distribuição (SUN et al., 2000). A massa molar média em massa $\left(\bar{M}_{w}\right)$ e a massa molar média em número $\left(\bar{M}_{n}\right)$, obtidas pela cromatografia de permeação em gel, alcançaram os valores de 3969 e 1133 Da, respectivamente. O valor encontrado para a polidispersão foi de 3,5 , resultado que aponta que a lignina de Pinus oocarpa possui distribuição de moléculas relativamente pouco dispersa, ou seja, suas massas molares estão compreendidas em um intervalo relativamente estreito.

A oxidação de ligninas com nitrobenzeno produz a vanilina como produto principal. Folhosas formam a vanilina e o seringaldeído, enquanto gramíneas, além desses dois aldeídos, produz o $p$-hidroxibenzaldeído. Na oxidação da LMM do Pinus oocarpa foram encontrados os compostos: $p$-hidroxibenzaldeído, vanilina e 4-hidróxi-3-metoxibenzóico. Não foi detectada a presença do seringaldeído e do ácido 4-hidróxi-3,5dimetoxibenzóico. Tal resultado confirma a natureza guaiacílica da lignina do Pinus oocarpa e que existem unidades aromáticas não metoxiladas na estrutura dessa macromolécula, como indicou a fórmula mínima $\left(\mathrm{C}_{9}\right)$.

A Figura 1 ilustra o espectro no infravermelho com transformada de Fourier (IVTF). O espectro mostrado é típico de ligninas. Portanto, o método utilizado na extração e purificação da lignina da madeira do Pinus oocarpa foi adequado e forneceu uma amostra que é representativa dessa macromolécula. As bandas a $1.268,17$ e $1.137,80 \mathrm{~cm}^{-1}$ indicam, também, que a lignina da madeira do Pinus oocarpa é do tipo guaiacila.

Na Figura 2, apresenta-se o espectro de RMN de ${ }^{13} \mathrm{C}$ da LMM-Ac. A análise do espectro ratifica as observações da espectroscopia no IVTF de que a lignina 
dessa madeira é do tipo guaiacila. Na Tabela 2 se encontram as atribuições de sinais característicos dessas unidades (NIMZe LUDEMANN, 1976; LIITIA et al., 2003).

O sinal devido ao carbono metoxílico (56,4 ppm) está bastante intenso, indicando que a abundância

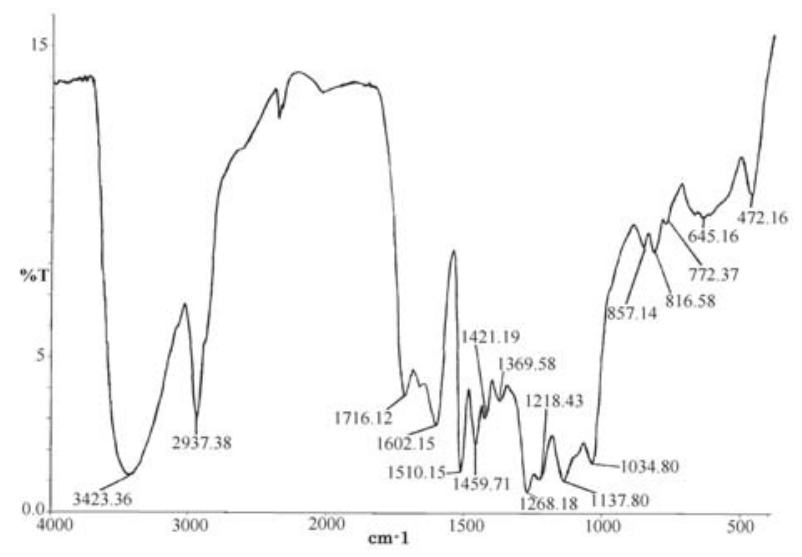

Figura 1 - Espectro no IVTF da lignina de madeira moída do Pinus oocarpa, em pastilha de $\mathrm{KBr} 1 \%$.

Figure 1 - FTIR spectrum of Pinus oocarpa $M W L$, in $1 \%$. $K B r$. desse grupo na macromolécula de lignina é elevada. Contaminantes alifáticos na lignina isolada são também evidentes nesse espectro, apresentando sinais de 21,3 a 30,0 ppm. As pequenas intensidades desses sinais evidenciam que a porcentagem desses contaminantes é pouco significativa.

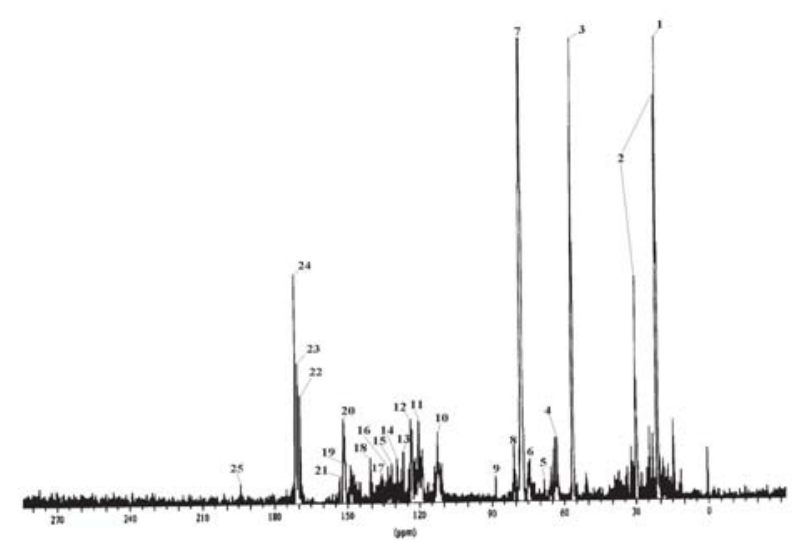

Figura 2 - Espectro de RMN de $13 \mathrm{C}$ da LMM acetilada do Pinus oocarpa.

Figure 2 - 13C NMR spectrum of acetylated MWL from Pinus oocarpa.

Tabela 2 - Atribuições dos sinais no espectro de RMN de 13 C da LMM acetilada do Pinus oocarpa Table 2 - Signals assignment in the 13C NMR spectrum of acetylated MWL from Pinus oocarpa

\begin{tabular}{|c|c|c|}
\hline Pico № & $\delta(\mathrm{ppm})$ & Atribuição \\
\hline 1 & 21,0 & $-\mathrm{CH}_{3}$ em grupo acetila \\
\hline 2 & 21,3 a 30,0 & $-\mathrm{CH}_{3}^{3}$ e $\mathrm{CH}_{2}$ em cadeias alifáticas saturadas \\
\hline 3 & 56,4 & $-\mathrm{OCH}_{3}^{3}$ \\
\hline 4 & 63,4 & $\mathrm{C} \gamma$ em unidades $\beta-\mathrm{O}-4, \mathrm{Cb}$ em unidades fenilcumaranas \\
\hline 5 & 67,5 & $\mathrm{C} \gamma$ em unidades fenilcumaranas \\
\hline 6 & 75,5 & $\mathrm{C} \alpha$ em unidades b-O-4 \\
\hline 7 & $77,0-78,0$ & Solvente $\left(\mathrm{CDCl}_{3}\right)$ \\
\hline 8 & 81,3 & $\mathrm{C} \beta$ em unidades $\beta-\mathrm{O}-4$ \\
\hline 9 & 89,6 & C $\alpha$ em unidades fenilcumaranas \\
\hline 10 & 112,2 & $\mathrm{C}_{2}$ em unidades guaiacílicas e $\mathrm{C}_{2}$ em unidades guaiacílicas acet. ( $\mathrm{G}-\mathrm{CH}(\mathrm{OAc})$-) \\
\hline 11 & 120,2 & $\mathrm{C}_{5}^{2}$ em $\beta$-aril éter, eterificada (forma eritro); $\mathrm{C}_{6} \mathrm{G}-\mathrm{CH}(\mathrm{OAc})-\beta$-aril éter \\
\hline $\begin{array}{l}12 \\
13\end{array}$ & $\begin{array}{l}123,2 \\
127,5\end{array}$ & $\begin{array}{l}\mathrm{C}_{5}^{5} \text { unidades guaiacílicas acet. (C } \alpha \text {-OAc e -OR); } \mathrm{C} \beta \text { em unidades guaiacílicas acet. } \\
\mathrm{C}_{2} / \mathrm{C}_{6} \text { de unidades p-hidroxifenílicas }\end{array}$ \\
\hline 14 & 128,0 & $\mathrm{C} \beta$ em unidades guaiacílicas $(\mathrm{G}-\mathrm{CH}=\mathrm{CH}-\mathrm{CHO})$ \\
\hline 15 & 131,5 & $\mathrm{C}_{1}$ em unidades guaiacílicas (eterificadas); $\mathrm{C}_{5}$ em unidades bifenílicas $\left(\mathrm{C}_{5}-\mathrm{C}_{5}\right)$ \\
\hline 16 & 132,6 & $\mathrm{C} \beta$ em cinamaldeído \\
\hline 17 & 135,7 & $\mathrm{C}_{4}$ em unidades $\mathrm{Se}-\mathrm{CH}(\mathrm{OAc})$ \\
\hline 18 & 140,0 & $\mathrm{C}_{1} \mathrm{e} \mathrm{C}_{4}$ em unidades G-CH-O-; $\mathrm{C}_{4}$ em unidades $\mathrm{G}-\mathrm{CH}(\mathrm{OAc})$ \\
\hline 19 & 147,1 & $\mathrm{C}_{4}$ em unidades guaiacílicas eterificadas $(\beta$-aril-éter, forma eritro) \\
\hline 20 & 151,4 & $\mathrm{C}_{3}^{4}$ unidades guaiacílicas acet. $(\mathrm{C} \alpha-\mathrm{OAc}) ; \mathrm{C}_{4}$ unidades guaiacílicas $(\mathrm{C} \alpha-\mathrm{OR}$ e -OAc) \\
\hline 21 & 152,4 & $\mathrm{C}_{3}^{3}$ em unidades guaiacílicas eterificadas; $\mathrm{C} \alpha \mathrm{em}$ unidades guaiacílicas \\
\hline 22 & 169,1 & $\mathrm{CO}$ em acetila aromática \\
\hline 23 & 170,0 & CO em acetila alifática secundária \\
\hline 24 & 171,1 & $\mathrm{CO}$ em acetila alifática primária \\
\hline 25 & 194,5 & $\alpha-\mathrm{CO}$ e $\gamma-\mathrm{CHO}$ \\
\hline
\end{tabular}

$\mathrm{G}=$ unidade guaiacílica, $\mathrm{Ac}=$ grupo acetila e $\mathrm{Se}=$ siringila eterificada. 
Na Figura 3, apresenta-se o espectro de RMN de ${ }^{1} \mathrm{H}$. As atribuições dos sinais que aparecem nesse espectro estão citadas na Tabela 3 (LUNDQUIST, 1979; RALPH et al., 1998).

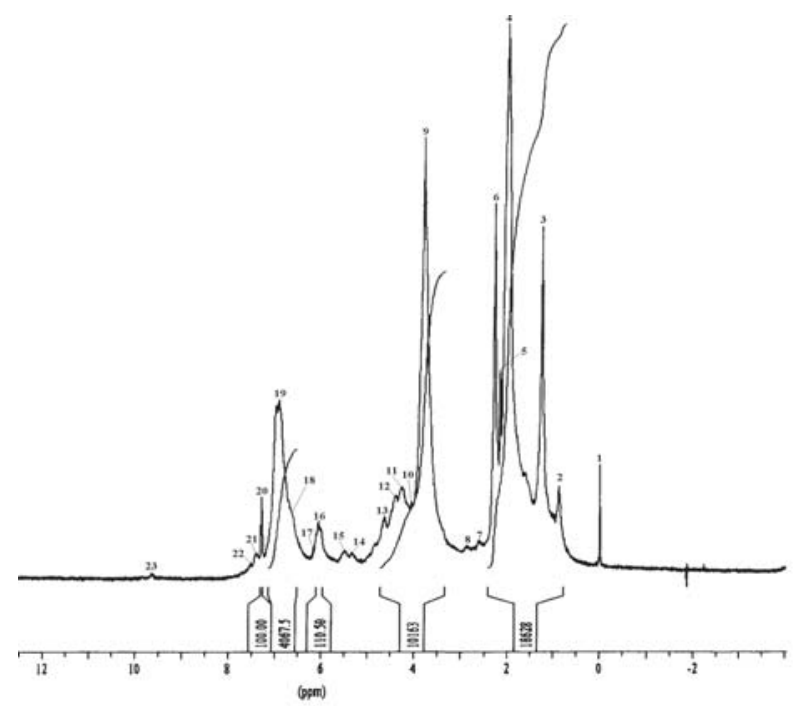

Figura 3 - Espectro de RMN de $1 \mathrm{H}$ da LMM acetilada do Pinus oocarpa.

Figure 3 - 1H NMR spectrum of acetylated MWL from Pinus oocarpa.
Informações importantes quanto aos grupos hidroxilas presentes na macromolécula de lignina podem ser obtidas do espectro de RMN de ${ }^{1} \mathrm{H}$. Os sinais a 2,02 ppm (prótons de acetato alifático) e a 2,16 e 2,29 ppm (prótons de acetatos aromáticos) são originários de hidroxilas alifáticas e fenólicas, respectivamente. De acordo com a intensidade desses sinais e conhecendo o valor da quantidade molar de hidroxilas totais na macromolécula de lignina $(0,92)$, calculou-o equivalente a 0,64 de hidroxila alifática e 0,28 de fenólicas, ou seja, em cada 100 unidades $C_{9}$ da lignina do Pinus oocarpa são encontradas 64 hidroxilas alifáticas e 28 hidroxilas fenólicas.

$\mathrm{O}$ espectro de RMN de ${ }^{1} \mathrm{H}$ mostra, ainda, que o sinal devido a átomos de hidrogênio metoxílicos a 3,81 ppm é relativamente intenso. Em contraposição, os prótons da cadeia lateral proveniente de unidades cinamílicas $(6,00-6,20 \mathrm{ppm})$ apresentam sinais pouco intensos, indicando que existe pouco desse tipo de subestrutura na macromolécula de lignina. O mesmo foi observado com relação aos sinais a 6,60 ppm que, provavelmente, são de prótons vinílicos, pois unidades siringílicas não foram detectadas. Os sinais de hidrogênio pouco intensos a 2,29; 2,$60 ; 2,80 ; 4,05$; 4,26; e 5,26 ppm indicaram a presença de subestruturas

Tabela 3 - Atribuições dos sinais no espectro de RMN de 13 C da LMM acetilada do Pinus oocarpa Table 3 - Signals assignment in the 13C NMR spectrum of acetylated MWL from Pinus oocarpa

\begin{tabular}{|c|c|c|}
\hline Pico № & $\delta(\mathrm{ppm})$ & Atribuição \\
\hline 1 & 0,0 & TMS \\
\hline 2 & 0,88 & $\left(-\mathrm{CH}_{2}-\right)$ Prótons de hidrocarbonetos alifáticos (contaminantes) \\
\hline 3 & 1,26 & $\left(-\mathrm{CH}_{2}^{-}\right)$Prótons de hidrocarbonetos alifáticos (contaminantes) \\
\hline 4 & 2,02 & $\left(\mathrm{CH}_{3}{ }^{2}\right)$ Prótons de acetato alifático \\
\hline 5 & 2,16 & $\left(\mathrm{CH}_{3}-\right)$ Prótons de acetato alifático ou fenólico \\
\hline 6 & 2,29 & $\left(\mathrm{CH}_{3}^{-}\right)$Prótons de acetato fenólico \\
\hline 7 & 2,60 & (H-) $\alpha$ de subestruturas $\beta-\beta$ do tipo isotaxiresinol e secoisolariciresinol (Figura 8 ) \\
\hline 8 & 2,80 & $\begin{array}{l}\text { B } \alpha \text { de subestruturas } \beta-\beta \text { do tipo isotaxiresinol e } \alpha_{1} \text { e } \alpha_{2} \text { de subestruturas do tipo secoisolariciresinol } \\
\text { (Figura } 8 \text { ) }\end{array}$ \\
\hline 9 & 3,82 & $\left(\mathrm{CH}_{3} \mathrm{O}_{-}\right)$Prótons em grupos metoxílicos \\
\hline 10 & 4,05 & $(\mathrm{H}-)^{3} \alpha$ de subestruturas isotaxiresinol; $(\mathrm{H}-) \gamma 1$ de subestruturas secoisolariciresinol \\
\hline 11 & 4,26 & $(\mathrm{H}-) \gamma$ em várias estruturas \\
\hline 12 & 4,40 & $(\mathrm{H}-) \gamma$ em subestruturas $\beta-\mathrm{O}-4$ (forma eritro) e subestruturas $\beta-5$ \\
\hline 13 & 4,63 & (H-) $\beta$ em subestruturas $\beta-\mathrm{O}-4$ (prótons metilênicos em unidades cinamílicas alcoólicas) \\
\hline 14 & 5,26 & $(\mathrm{H}-) \alpha$ em subestruturas fenicumarama bifenílica (Figura 8) \\
\hline 15 & 5,48 & $\begin{array}{l}(\mathrm{H}-) \alpha \text { em subestruturas } \beta-5 ;(\mathrm{H}-) \alpha \text { em benzilaril éteres não-cíclicos, }(\mathrm{H}-) \beta \text { em } \\
\text { 2-arilpropilfenonas }\end{array}$ \\
\hline 16 & 6,05 & $\begin{array}{l}\text { (H-) } \alpha \text { em estruturas } \beta \text {-O-4 e } \beta \text { - } 1 \text {; Hb em unidades cinamílicas alcoólicas; prótons aromáticos (de unidades } \\
\text { guaiacílicas) }\end{array}$ \\
\hline 17 & 6,18 & $(\mathrm{H}-) \beta$ de subestruturas vinílicas eterificadas ou não \\
\hline 18 & 6,60 & Prótons aromáticos de unidades siringílicas, alguns prótons vinílicos \\
\hline 19 & 6,93 & Prótons aromáticos de unidades guaiacílicas, alguns prótons vinílicos \\
\hline 20 & 7,29 & Clorofórmio (solvente) \\
\hline 21 & 7,40 & Prótons aromáticos de subestruturas do tipo benzaldeído \\
\hline 22 & 7,50 & Prótons aromáticos orto a grupos carbonílicos \\
\hline 23 & 9,70 & Prótons formila em subestruturas do tipo cinamaldeído e benzaldeído \\
\hline
\end{tabular}

R. Árvore, Viçosa-MG, v.29, n.3, p.471-478, 2005 
complexas, como: isotaxiresinol, secoisolariciresinol e 1-(3,4-dimetoxifenil)-2-(4-carboximetilfnóxi)-propan1,3-diol, que podem estar presentes na macromolécula dessa lignina (LUNDQUIST, 1979; RALPH et al., 1998).

\section{CONCLUSÕES}

Os estudos da macromolécula de LMM do Pinus oocarpa evidenciaram que ela é uma lignina típica de coníferas, cuja fórmula mínima $\mathrm{C}_{9}$ pode ser expressa por $\mathrm{C}_{9} \mathrm{H}_{9,2} \mathrm{O}_{2,6}\left(\mathrm{OCH}_{3}\right)_{0,8}$, o que equivale a uma massa molar de $183,6 \mathrm{Da}$. Suas massas molares relativas $\left(\bar{M}_{w}\right)$ e $\left(\bar{M}_{n}\right)$ são 3969 e $1133 \mathrm{Da}$, respectivamente.

\section{AGRADECIMENTOS}

Os autores agradecem ao Instituto de Química da Universidade Federal de Uberlândia (IQUFU), pelo suporte durante as experiências; e à Fundação de Apoio à Pesquisa de Minas Gerais (FAPEMIG), pela bolsa de Iniciação Científica.

\section{REFERÊNCIAS BIBLIOGRÁFICAS}

CHEN, C.-L. Lignins: occurrence in woody tissues, isolation, reactions, and structure. In: LEWIN, M.; GOLDSTEIN, I. S. Wood structure and composition. New York: Marcel Decker, 1991.p. 183-261.

CHEN, C.-L. Nitrobenzene and cupric oxide oxidations. In: DENCE, C. W.; LIN, S. Y.; TIMELL, T. E. Methods in Lignin Chemistry. New York: Springer-Verlag, 1992a. p. 301-321.

CHEN, L.-C. Functional Group Analysis. In: DENCE, C. W.; LIN, S. Y.; TIMELL, T. E.

Methods in Lignin Chemistry. New York: Springer-Verlag, 1992b. p. 407-422.

COLOM, X. et al. Structural analysis of photodegraded wood by means of FTIR spectroscopy. Polymer Degradation and Stability, v.80, n. 3, p. 543-549, 2003.

FREUDENBERG, K.; NEISH, A. C. Constitution and biosynthesis of lignin. BerlinHeidelberg-New York: Springer Verlag, 1968. 120 p.
GUERRA, A.; MENDONÇA, R.; FERRAZ, A. Molecular weight distribution of wood components extracted from Pinus taeda biotreated by Ceriporiopsis subvermispora. Enzyme and Microbial Technology, v.33, p. 12-18, 2003.

KILPELAINEN, I. et al. Application of 2dimensional NMR-spectroscopy to wood lignin structure determination and identification of some minor structural units of hardwood and softwood lignins. Journal of Agricultural and Food Chemistry, v. 42, n. 12, p. 2790-2794, 1994.

KRÖGER. C.; DARSOW, G.; FUHR, K. Physikalisch-chemische Eigenschaften von Braunkohlen und Braunkohlenkomponenten. v. Die Carbonylgruppen und Äther Sauerstoff Bestimmung. Erdöl und Kohle Erdgas Petrochemie, v. 18, n. 9, p. 701-710, 1965.

LIITIA, T. M. et al. Analysis of technical lignins by two- and three-dimensional NMR spectroscopy. Journal of Agricultural and Food Chemistry, v.51, n. 8, p. 21362143, 2003.

LIMA, A.F.; JARÁ, E. R. P.; ALFONSO, V. A. Madeira como matéria-prima para fabricação de pasta celulósica. In: PHILIPP, P.; D’ ALMEIDA, M. L.O. Celulose e papel: tecnologia de fabricação da pasta celulósica. 2. ed. São Paulo: IPT, 1988. p.129-167.

LUNDQUIST, K. NMR studies of lignins. 2. Interpretation of the ${ }^{1} \mathrm{H}$ NMR spectrum of acetylated birch lignin. Acta Chemistry Scandinavian, v. B33, p. 27-30, 1979.

LUNDQUIST, K. Isolation and Purification. In: DENCE, C. W.; LIN, S. Y.; TIMELL, T. E. Methods in Lignin Chemistry. New York: Springer-Verlag, 1992. p. 63-17.

MORAIS, S. A. L. Contribuição ao estudo químico e espectroscópico da lignina de madeira moída do Eucalyptus grandis: isolamento, quantificação e análise estrutural.1992. 175 f. Doutorado (Doutor em Ciências - Química Orgânica). Universidade Federal de Minas Gerais, Belo Horizonte, 1992.

R. Árvore, Viçosa-MG, v.29, n.3, p.471-478, 2005 
MORAIS, S. A. L.; PILÓ-VELOSO, D.;

NASCIMENTO, E. A. Isolamento e análise estrutural de ligninas. Química Nova, v. 16, n. 5, p. 435-948, 1993.

NADA, A. A. M. A. et al. Infrared spectroscopy of some treated lignins. Polymer

Degradation and Stability, v. 62, n.1, p.157-163, 1998.

NIMZ, H. H.; LUDEMANN, H. D. ${ }^{13} \mathrm{C}$ NMRSpectra of Lignins. 6. Lignin and DHP Acetates. Holzforschung, v. 30, n. 2, p. 33-40, 1976.

PHILIPP, P.; D’ ALMEIDA, M. L.O. Celulose e papel: tecnologia de fabricação da pasta celulósica. 2. ed. São Paulo: IPT, 1988. 964 p.
RALPH, S. A. et al. NMR Database of Lignin and Cell Wall Model

Compounds. US Forest Products Laboratory USDA Forest Service One Gifford Pinchot. 1998. Disponível em: http://www.dfrc.ars.usda.gov/ software.html. Acesso em: 25 de maio de 2002.

SUN, R. et al. Characterization of lignins from wheat straw by alkaline peroxide treatment.

Polymer Degradation and Stability, v.67, p. 101-109, 2000.

ZAKIS, G. F.; JOYCE, T.; BREZNY, R. The functional analysis of lignins and their derivatives. Atlanta: TAPPI Press, 1994. $94 \mathrm{p}$. 